\title{
Primary appendicitis epiploicae mimicking acute appendicitis
}

\author{
A.J. McMahon and D.T. Hansell \\ Department of Surgery, Victoria Infirmary, Glasgow, UK.
}

\begin{abstract}
Summary: We report two cases of primary appendicitis epiploicae, caused by torsion, whose presentation mimicked acute appendicitis.
\end{abstract}

\section{Introduction}

Primary appendicitis epiploicae is a rare condition. ${ }^{1}$ There have been no reports of primary appendicitis epiploicae in the English literature in the last 30 years. Recognition of the condition is important because, if untreated, it may be complicated by intestinal obstruction or peritonitis. ${ }^{1,2}$ When these complications occur, there is a high mortality. ${ }^{2} \mathrm{We}$ present two case reports.

\section{Case reports}

\section{Case 1}

A 32 year old man presented with a 5-day history of colicky central abdominal pain moving to the right iliac fossa. Pain was worsened by movement and coughing. There was no anorexia, nausea, vomiting or systemic upset. He had a past history of ulcerative colitis. On examination of his abdomen, there was localized tenderness, rebound, and guarding in the right iliac fossa. He was apyrexial and the white cell count was 10.9 cells $\times 10^{9} / 1$. A presumptive diagnosis of acute appendicitis was made.

At laparotomy there was a moderate amount of free turbid fluid in the abdomen (culture of which was sterile). The vermiform appendix appeared normal. A $2 \mathrm{~cm}$ diameter irregular firm black lesion was found on the anterior wall of the caecum. This was excited by blunt dissection. The appearances were consistent with an appendix epiploicae which

Correspondence: A.J. McMahon M.B., Ch.B., 42 Airlie Street, Hyndland, Glasgow G12 9TR, UK Accepted: 30 June 1988 had become torted and undergone haemorrhagic infarction. Routine appendicectomy was carried out. Histology of the appendix epiploicae showed severe congestion and fat necrosis in the lesion and associated peritonitis. Histology of the vermiform appendix showed mucosal changes compatible with previously known diagnosis of ulcerative colitis. Recovery was uneventful.

\section{Case 2}

A 28 year old woman presented with a 4-day history of progressive constant abdominal pain in the right iliac fossa. The pain was worse on moving, coughing and straining. She was also flushed and shivery. There was no anorexia, nausea, vomiting, diarrhoea or systemic upset. She had a low grade pyrexia. On abdominal examination there was a mass in the right iliac fossa with localized tenderness, guarding and rebound. Bowel sounds were normal. Rectal and vaginal examination were normal. The general practitioner, gynaecologist, and surgeon respectively had made a diagnosis of torsion of an ovarian cyst, abdominal wall haematoma, and acute appendicitis with an inflammatory mass.

At laparotomy the vermiform appendix, right ovary and uterus were found to be normal. There was a mass in the right iliac fossa consisting of loops of small bowel and inflamed omentum wrapped around the caecum. Small bowel and omentum were freed by blunt dissection, revealing a firm, plum-coloured lesion ( $8 \mathrm{~cm}$ diameter) on a pedicle from the caecum (Figure 1). A malignant process was initially suspected and frozen section was carried out. This showed that it was an appen-

(C) The Fellowship of Postgraduate Medicine, 1988 


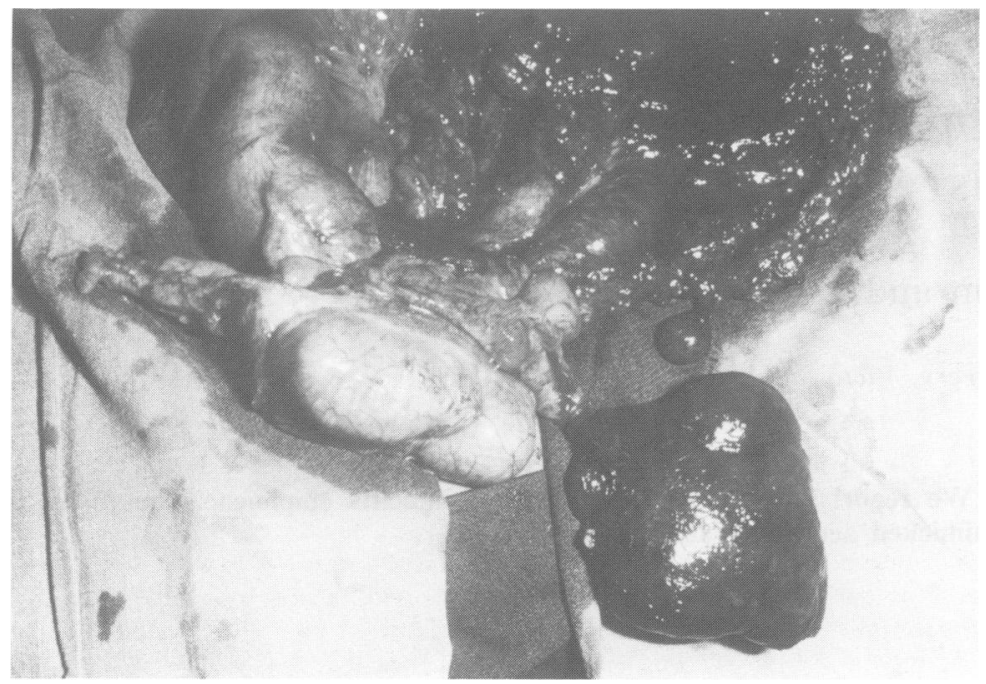

Figure 1 An appendix epiploicae, arising on a narrow pedicle from the caecum, which had undergone torsion and haemorrhagic infarction.

dix epiploicae which had torted and undergone haemorrhagic infarction. This was excised and definitive histology showed adipose tissue which had undergone infarction with secondary haemorrhage. Inflammatory changes were noted within the walls of deep vessels. Histology of the appendix, which was removed routinely, showed old appendicitis. Postoperative recovery was uneventful.

\section{Discussion}

Appendicitis epiploicae is a rare condition. The relative incidence compared to acute vermiform appendicitis is $0.2 \% .^{3}$ The largest reported series in the English language is 13 cases encountered over a 14 year period. ${ }^{2}$ The only such case report ${ }^{4}$ in the last 30 years may have been secondary to diverticulitis, as there was an underlying diverticulum and there was no torsion. Also the lesion was not excised and there was no histology performed. In this decade, there have been only three individual case reports in the non-English literature, 5.6 and one series of 28 cases encountered over a 20 year period. ${ }^{7}$

Appendices epiploicae are fat-filled, serosacovered appendages of the colon present in two rows with a total of about one hundred. ${ }^{8}$ Most cases of appendicitis epiploicae are caused by torsion which comprises or strangulates the blood supply. ${ }^{9}$ The actual twist may not always be demonstrated at surgery. There may be varying degrees of torsion from sudden complete torsion to insidious twisting, which will give rise respectively to inflammatory changes and symptoms from acute to chronic. $^{10} \mathrm{~A}$ number of aetiological factors which might predispose to torsion have been postwe lated. There is usually a narrow base to the appen dix epiploicae, allowing it to rotate. Since a large percentage of the cases reported have been overweight, ${ }^{11}$ it is likely that obesity plays a part in the pathogenesis. Appendices epiploicae, hypertrophied as a result of obesity, are more likely to swing freely. Some cases of appendicitis epiploicae present in an inguinal or femoral hernia: torsion may be explained by the appendix twisting on entrance to the hernial ring during an episode of coughing. ${ }^{2}$

The site of the colon most commonly affected is the sigmoid and caecum, but it may occur on any part. It occurs predominantly in the age range 20 50 years. The only report in a child was of an extremely obese 12 year old. ${ }^{1}$ Although appendices epiploicae are first formed at 4 months gestation, they remain rudimentary until adult life. ${ }^{8}$ While in a review of the literature there was an equal incidence between the sexes, ${ }^{2}$ Szunyogh et al. found a female/male ratio of $5: 1$ in their series of $28 \%$ patients. $^{7}$

The most common presenting symptom is acute moderate to severe lower abdominal pain, more often on the left than on the right. It may be colicky or constant in nature. The pain has usually been present for less than a week (two thirds of $\stackrel{\oplus}{\rightarrow}$ patients), but in some it may have been present 
intermittently over several months. Vomiting occurs in a third of patients, and nausea alone in a sixth. Bowel habit is rarely altered. There is usually localized tenderness and guarding, but generalized tenderness or rebound tenderness are unusual. An abdominal mass is palpable in one third of cases. The temperature and white cell count are usually only marginally elevated, and often normal.

There are no pathognomonic features of primary appendicitis epiploicae. The most commonly made preoperative diagnoses are acute vermiform appendicitis $(60 \%)$, diverticulitis $(13 \%)$, torsion of an ovarian cyst $(6 \%)$, or cholecystitis $(3 \%){ }^{1}$ A correct preoperative diagnosis is rarely made. ${ }^{12}$ In some cases, primary appendicitis epiploicae may be completely asymptomatic, and the lesion may be encountered by the surgeon while performing a laparotomy for another condition. In this situation the lesion may be confused with a neoplastic process $^{1}$ (as in our second case report) and a frozen section is necessary to reach the diagnosis.

\section{References}

1. Lynn, T.E., Docherty, M.B. \& Waugh, J.M. A clinicopathological study of the epiploic appendages. Surg Gynec Obstet 1956, 103: 423-433.

2. Fieber, S.S. \& Forman, J. Appendices epiploicae: clinical and pathological considerations: report of three cases and statistical analysis on one hundred and five cases. Arch Surg 1953, 66: 329-338.

3. Bearse, C. Primal affections of appendices epiploicae. Am J Dig Dis 1952, 19: 22-24.

4. Daar, A.S. Acute primary epiploic appendagitis. $\mathrm{Br} J$ Clin Pract 1981, 35: 333-335.

5. Nurmukhamedov, R.M., Nadzhimitdinov, N.N., Makhmudov, Z.U. \& Uralov, M.M. Lesions of the greater omentum and fatty appendices. Khirurgia (Mosk) 1979, 10: 49-51.

6. Ditlevsen, A.S. \& Hansen, C. Appendicitis epiploicae. Ugeskr Laeger 1985, 147 (43): 3411.

7. Szunyogh, L. \& Palla, G. Torsion of the epiploic appendices in 28 cases. Orv Hetil 1982; 123 (48): 2953-2955.
In most untreated cases, the acute inflammatory phase is followed by aseptic necrosis with subsequent exfoliation and healing. However, the inflamed appendix epiploicae may become adherent to loops of bowel or mesentery, causing intestinal obstruction, usually of the small bowel. This occurs in $10 \%$ of cases and is associated with a high mortality. ${ }^{2}$ Rarely, secondary infection of an infarcted appendix epiploicae may cause an intraabdominal abscess or generalized peritonitis. ${ }^{13}$

The treatment of choice for primary appendicitis epiploicae consists of simple ligation and excision. ${ }^{4}$ It has been noted that mesenteric blood vessels loop into the base of appendices epiploicae before supplying the adjacent bowel wall. ${ }^{8,14}$ However, no cases of compromised viability of the intestinal wall have been reported as a result of excision of an appendix epiploicae. If an underlying colonic diverticulum is accidentally transected, the stump should be oversewn and inverted.

8. Pines, B., Rabinovitch, J. \& Biller, S.B. Primary torsion and infarction of the appendices epiploicae Arch Surg 1941, 42: 775-787.

9. Hunt, V.C. Torsion of appendices epiploicae. Ann Surg 1919; 69: 31.

10. Upham, R. \& McGrattan, V. Torsion of appendices? epiploicae: Review of the literature and report of one case. Bull New York M Coll 1940, 3: 240.

11. Giffin, H.M., McManamy, E.P. \& Waugh, J.M. Surgical significance of epiploic appendages. Arch Surg 1942, 45: 351.

12. Eliason, E.L. \& Johnson, J. Primary acute epiploitis. Surgery 1939, 6: 68 .

13. Patterson, D.C. Appendices epiploicae and their surgical significance with a report of three cases. $N$ Engl J Med 1933, 209, 1255.

14. Ross, J.A. Vascular loops in the appendices epiploicae: their anatomy and surgical significance, with a review of the surgical pathology of appendices epiploicae. Br J Surg 1950, 37: 464-466. 